УДК [616.361/.366+616.37-002]-089-072.1

DOI 10.11603/2414-4533.2019.4.10704

(ОВ. И. ЛУПАЛЬЦОВ, А. И. ЯГНЮК, Н. Н. СКАЛИЙ, М. С. КОТОВЩИКОВ

Харьковский национальный медицинский университет

\title{
Панкреатобилиарная манометрия в профилактике постманипуляционного панкреатита после эндоскопической ретроградной холангиопанкреатографии
}

\begin{abstract}
Цель работы: снижение частоты возникновения острого постманипуляционного панкреатита за счёт использования панкреатобилиарной манометрии.

Материалы и методы. Предложено устройство, регистрирующее давление в общем панкреатическом протоке при выполнении эндоскопической ретроградной холангиопанкреатографии (ЭРХПГ), которое в режиме реального времени с помощью графической кривой отображает давление в панкреатическом протоке. Так, при резком “пиковом” подъеме кривой ситуацию расценивали как повышение давления в панкреатическом протоке, что являлось показанием к введению контраста с меньшей объемной скоростью и проведению противопанкреатической терапии. Эта методика была использована у 128 пациентов с 2013 по 2018 гг.

Результаты исследований и их обсуждение. У больных первой группы острый постманипуляционный панкреатит развился у 21,8 \%, тогда как у пациентов второй группы, у которых профилактические мероприятия острого постманипуляционного панкреатита проводились тотчас после выявления его признаков - у 6,3 \%. Клинические симптомы острого панкреатита и амилолитическая активность крови в группе, где применялись методики манометрического контроля, наблюдались реже, чем в группе сравнения.

Использование разработанных методик манометрического контроля во время лечебно-диагностических манипуляций на фатеровом сосочке достоверно снижает риск возникновения острого постманипуляционного панкреатита.
\end{abstract}

Ключевые слова: острый постманипуляционный панкреатит; эндоскопическая ретроградная панкреатохолангиография; панкреатобилиарная манометрия.

Постановка проблемы и анализ последних исследований и публикаций. Эндоскопические чреспапиллярные вмешательства у пациентов с осложненными формами желчнокаменной болезни давно входят в стандарт лечения данной патологии, эффективность при их применении позволяет достичь приемлемых результатов в подавляющем большинстве случаев. Однако накопленный опыт показывает, что избежать осложнений эндоскопической ретроградной холангиопанкреатографии (ЭРХПГ) и эндоскопической папиллосфинктеротомии (ЭПСТ) не всегда удается, даже если они выполняются опытными специалистами $[1,2,3]$.

Тяжелым осложнением является острый постманипуляционный панкреатит (ОПМП) развивающийся в среднем в 25-30 \% случаев, отличающийся быстрым развитием и тяжелым течением $[7,8]$, с летальностью, по данным различных авторов, до $13 \%[6,9,10]$.

Термин ОПМП известен более 20 лет и, согласно международной классификации Yoon $\mathrm{H}$. и соавт. [8], обозначает клиническое проявление панкреатита, сопровождающегося повышением уровня амилазы более чем в три раза, развивающегося, как правило, в течение 24 часов после манипуляций на большом сосочке двенадцатиперст- ной кишки (БСДПК), требующее наблюдения в стационаре либо продления госпитализации.

Позже Parsi М. и соавт. (2014) [6] внесли изменения в определение ОПМП, обозначив его как появившуюся или усиливающуюся боль в животе, сопровождающуюся повышением уровня амилазы и/или липазы более трех норм, развивающуюся в ближайшие 24 часа после эндоскопической интервенции и требующая госпитализации или продления ее срока на 2-3 дня [9, 10].

По данным Benjaminov F. (2014), частота возникновения постманипуляционных осложнений в центрах, где производится менее 200 ЭРХПГ в год, в 3 раза выше, чем там, где в год выполняется более 200 манипуляций [4].

Причинами, приводящими к развитию ОПМП, большинство авторов считают механические и термические повреждения устья или дистальной части главного панкреатического протока (ГПП); осмотическую и гидродинамическую травму ацинусов поджелудочной железы при внутрипротоковом введении контрастного вещества; нарушение целостности слизистой оболочки ГПП; контактное инфицирование системы ГПП; компрессионное повреждение паренхимы поджелудочной железы при проведении агрессивной тракции конкрементов. 
Для оценки возможностей медикаментозной профилактики реактивного постманипуляционного панкреатита во всем мире проводится большое количество контролируемых клинических исследований, связанных с использованием широкого спектра лекарственных препаратов (включая соматостатин, октреотид и другие антиферментные препараты).

В последнее время в зарубежной и отечественной литературе $[3,5,8]$ стали широко освещаться вопросы превентивного протезирования главного панкреатического протока как с целью профилактики развития ОПМП, так и в качестве одного из методов его лечения, особенно у пациентов с высоким риском развития подобного осложнения.

Часть авторов предлагает использование нестероидных противовоспалительных препаратов перед проведением ЭРПХГ, что также, по их мнению, позволяет снизить частоту развития острого постманипуляционного панкреатиата.

Таким образом, несмотря на актуальность, и востребованность выполнения ЭРХПГ, расширение её использования сдерживается пониманием риска постманипуляционных осложнений, главным из которых остается развитие ОПМП.

Цель работы: снижение частоты возникновения острого постманипуляционного панкреатита за счёт использования панкреатобилиарной манометрии.

Материалы и методы. В исследование включено 244 больных, которым с 2013 по 2018 год выполнялась ЭРХПГ и транспапиллярные вмешательства для ликвидации обтурационной желтухи, с повышением уровня билирубина свыше 35 мкмоль/л, обусловленной желчнокаменной болезнью, осложненной холедохолитиазом, стенозирующим паппиллитом и рубцовыми стриктурами терминального отдела холедоха.

В качестве основного скринингового метода диагностики ЖКБ, обуславливающего желтуху, использовали ультразвуковое исследование (УЗИ). В зависимости от конкретных условий, размера конкрементов, тяжести состояния больного выполнялись различные методы декомпрессии: папиллотомия, папиллодилатация, баллонная дилатация БСДПК.

Средний возраст пациентов составил $(53,43 \pm 2,14)$ лет, мужчин было 142 (58,2 \%), женщин - 102 (41,8 \%). Распределение больных по возрастным группам соответствовало закону нормального распределения ( $<0,05)$, что делало возможным использования параметрических методов статистики.

Проводился анализ физикальных данных, выполнялся забор клинических и биохимических анализов, а также УЗИ при клинических проявлениях ОПМП.

Анализируемые пациенты были разделены на две группы. В первую группу включены 116 больных с нарушением билиарной проходимости, которым выполнялась диагностическая и лечебная ЭРХПГ без измерения давления в главном панкреатическом протоке и ранних лечебно-профилактических мероприятий при развитии ОПМП из-за поздней его диагностики.

Вторую группу составили 128 больных, у которых профилактические мероприятия острого постманипуляционного панкреатита проводились тотчас после выявления его признаков с помощью предложенного нами устройства регистрирующего давление в общем панкреатическом протоке (патент на полезную модель UA104992, 2016), представляющего собой шприц для введения контраста с системой тройников, в которую интегрирован датчик MPX 5010 DP фирмы Motorola.

При введении шприцем контраста датчиком определялось давление, которое в режиме реального времени беспроводным интерфейсом Bluetooth передавалось на компьютер, где интерпретировалось графической кривой различной конфигурации, зависящей от давления в гепатикохоледохе.

Так, при резком “пиковом” подъеме кривой ситуацию расценивали как повышение давления в панкреатическом протоке, скорость введения контраста уменьшали, а при реакции поджелудочной железы, проявляющейся симптоматикой панкреатита, чаще триадой Мондора, проводили лечебнопрофилактические мероприятиям ОПМП.

При выполнении ЭРХПГ, когда имелись показания для билиарной декомпрессии, выполнялась эндоскопическая папиллосфинктеротомия или баллонная дилатация с проведением санации желчевыводящих протоков до стабилизации состояния и нормализации уровня билирубина. Состояние больных после выполнения эндоскопического лечения оценивали клинически, по данным УЗИ и клинико-биохимических анализов.

Результаты исследований и их обсуждение. При выполнении ЭРХПГ у больных выявляли наличие стеноза БСДПК или наличие конкрементов в холедохе, которые требовали выполнения ЭПСТ. Эндоскопическая папиллосфинктеротомия выполнена у 30 (23,4 \%) больных первой группы и 36 (31,0 \%) больных второй группы.

При выполнении ЭРХПГ стандартным способом нами, зачастую, отмечался рефлюкс контраста в панкреатический проток, обуславливающий развитие ОПМП, что в значительной степени и определяет неудовлетворительные результаты 
при выполнении этого исследования. При применении предложенного нами метода такой рефлюкс наблюдался реже из-за оценки графической кривой до его развития. Суть аппаратного преимущества контроля развития рефлюкса состоит в ранней регистрации повышения давления в панкреатическом протоке, отображающееся графической кривой различной конфигурации. "Пикообразный” подъем кривой сигнализирует о повышении давления в панкреатическом протоке и если продолжить введение контраста с той же объемной скоростью, то высок риск развития постманипуляционного острого панкреатита. Своевременное снижение объемной скорости введения контраста предупреждает гипертензию в главном панкре- атическом протоке, чем и объясняется снижение случаев возникновения ОПМП.

В таблице представлена частота развития клинических симптомов ОПМП и явлений гиперамилаземии после выполнения транспапиллярных вмешательств по предлагаемой нами методике.

Таким образом, анализ проведенных нами исследований показал, что у больных первой группы ОПМП развился у 21,8 \%, тогда как у пациентов второй группы только у 6,3 \%. По представленным данным видно, что клинические симптомы панкреатита и повышение амилолитической активности крови в группе, где применялись методики манометрического контроля, наблюдались реже, чем в группе сравнения.

Таблица. Частота развития гиперамилаземии и клиники острого постманипуляционного панкреатита после выполнения эндоскопической ретроградной холангиопанкреатографии традиционной и модифицированной методикой

\begin{tabular}{|c|c|c|c|c|c|}
\hline & & & Гетоди & ирова & \\
\hline Оцениваемые по & & & & мод & ная \\
\hline & & $\mathrm{n}$ & $\%$ & $\mathrm{n}$ & $\%$ \\
\hline Болевой синдром & есть & 28 & 21,9 & 8 & 6,9 \\
\hline & нет & 100 & 78,1 & 112 & 93,1 \\
\hline Рвота & есть & 14 & 18,8 & 6 & 5,2 \\
\hline & нет & 104 & 81,2 & 110 & 94,8 \\
\hline Парез кишечника & есть & 26 & 20,3 & 6 & 5,2 \\
\hline & нет & 102 & 79,7 & 110 & 94,8 \\
\hline Симптом Куллена & есть & 16 & 12,5 & 2 & 1,7 \\
\hline & нет & 116 & 87,5 & 114 & 98,3 \\
\hline Симпом Мейо-Робсона & есть & 4 & 3,1 & - & - \\
\hline & нет & 124 & 96,9 & - & - \\
\hline Гиперамилаземия & есть & 36 & 28,1 & 12 & 7,4 \\
\hline & нет & 92 & 71,9 & 108 & 89,6 \\
\hline Всего & & & & & \\
\hline
\end{tabular}

Примечание: статистическая значимость между группами достоверна, p <0,05.

Выводы. Использование разработанных методик манометрического контроля давления в гепатикохоледохе во время лечебно-диагностических манипуляций на фатеровом сосочке, достоверно снижает риск возникновения острого постманипуляционного панкреатита. 


\section{ОРИГІНАЛЬНІ ДОСЛІДЖЕННЯ}

\section{СПИСОК ЛИТЕРАТУРЫ}

1. Скалій М. М. Вдосконалення мініннвазивних методів діагностики і лікування малосимптомних форм порушення прохідності термінального відділу холедоха: автореферат ... дис. канд. мед. наук: 14.01.03 - хірургія / М. М. Скалій; МОЗ України, Харків. мед. акад. післядипл. освіти. - Х., 2016. - 22 с.

2. Шадрін О. В. Вибір ендоскопічних методів декомпресії жовчовивідних протоків у хворих з гострим біліарним панкреатитом: автореферат. дис. ... канд. мед. наук: 14.01.03 - хірургія / О. В. Шадрін; МОЗ України, Харків. мед. акад. післядипл. освіти. - Х., 2011. - 17 с.

3. Хирургическая тактика лечения холедохолитиаза, осложненного механической желтухой, у больных с измененной анатомией билиодуоденальной области / С. Г. Шаповальянц, Т. Б. Ардасенов, Е. Д. Федоров [и др.] // Хирургия. - 2011. № 10 . - C. 35-38.

4. Benjaminov F. Effects of age and cholecystectomy on common bile duct diameter as measured by endoscopic ultrasonography / F. Benjaminov, G. Leichtman, T. Naftali // Surg. Endosc. - 2013. - Vol. 27. - P. 303-307.

5. Intraoperative endoscopic retrograde cholangiopancreatography: A useful tool in the hands of the hepatobiliary surgeon / A. el Nakeeb, A. M. Sultan, E. Hamdy [et al.] //World

\section{REFERENCES}

1. Skalii M.M. (2016). Vdoskonalennia miniinvazyvnykh metodiv diahnostyky i likuvannia malosymptomnykh form porushennia prokhidnosti kholedokha [Improvement of minimally invasive diagnostic and treatment methods oligosymptomatic forms impaired patency of the terminal part of the common bile duct]. Candidate's thesis. Kharkiv: Medical Academy of Postgraduate Education of Ukraine [in Ukrainian].

2. Shadrin, O.V. (2016). Vybir endoskopichnykh metodiv dekompresii zhovchovyvidnykh protokiv u khvorykh z hostrym biliarnym pankreatytom [Choice of endoscopic methods of decompression of bile ducts in patients with acute biliary pancreatitis]. Candidate's thesis. Kharkiv [in Ukrainian].

3. Shapovalants, S.G., Ardasenov, B., \& Fedorov, E.D. (2011). Khirurgicheskaya taktika lecheniya kholedokholigiaza, oslozhnennogo mekhanicheskoy zheltukhoy u bolnykh s izmenennoy anatomiyey biliodoedenalnoy oblasti [Surgical tactics for the treatment of choledocholigiasis complicated by obstructive jaundice in patients with altered anatomy of the bilioduodenal region]. Khirurgiya - Surgery, 10, 35-38 [in Russian].

4. Benjaminov, F., Leichtman, G., \& Naftali, T. (2013). Effects of age and cholecystectomy on common bile duct diameter as measured by endoscopic ultrasonography. Surg. Endosc., 27,
Journal of Gastroenterology: WJG. - 2015. - Vol. 21, No. 2. P. 609-615.

6. Diagnostic and therapeutic cholangiopancreatoscopy: performance of a new digital cholangioscope / M. A. Parsi, S. Jang, M. Sanaka [et al.] // Gastrointest. Endosc. - 2014. Vol. 79. - P. 936-942.

7. Trikudanathan G. Endoscopic management of difficult common bile duct stones / G. Trikudanathan, U. Navaneethan, M. A. Parsi // World J. Gastroenterol. - 2013. - Vol. 19. - P. 165173.

8. Endoscopic papillary large balloon dilation for the management of recurrent difficult bile duct stones after previous endoscopic sphincterotomy / H. G. Yoon, J. H. Moon, H. J. Choi [et al.] // Dig. Endosc. - 2014. - Vol. 26. - P. 259-263.

9. Risk factors associated with residual stones in common bile duct via $\mathrm{T}$ tube cholangiography after common bile duct exploration. Mubarak / J. F. Zhang, Z. Q. Du, Q. Lu [et al.] // Medicine. - 2015. - Vol. 94, No. 26. - P. e1043.

10. Zhao H. C. Meta-analysis comparison of endoscopic papillary balloon dilatation and endoscopic sphincteropapillotomy / H. C. Zhao, L. He, D. C. Zhou [et al.] // World J. Gastroenterol. 2013. - Vol. 19. - P. 3883-3891.

303-307.

5. El Nakeeb, A., Sultan, A.M., \& Hamdy, E. (2015). Intraoperative endoscopic retrograde cholangio-pancreatography: A useful tool in the hands of the hepatobiliary surgeon. World Journal of Gastroenterology: WJG, 21 (2), 609-615.

6. Parsi, M.A., Jang, S., \& Sanaka, M. (2014). Diagnostic and therapeutic cholangiopancreatoscopy: performance of a new digital cholangioscope. Gastrointest. Endosc., 79, 936-942.

7. Trikudanathan, G., Navaneethan, U., \& Parsi, M.A. (2013). Endoscopic management of difficult common bile duct stones. World J. Gastroenterol., 19, 165-173.

8. Yoon, H.G., Moon, J.H., \& Choi, H.J. (2014). Endoscopic papillary large balloon dilation for the management of recurrent difficult bile duct stones after previous endoscopic sphincterotomy. Dig. Endosc., 26, 259-263.

9. Zhang, J.F., Zhang, Z.Q., Du, Q., \& Lu, J.F. (2015). Risk factors associated with residual stones in common bile duct via $\mathrm{T}$ tube cholangiography after common bile duct exploration. Medicine, 94 (26), e1043.

10. Zhao, H.C., He, L., \& Zhou, D.C. (2013). Meta-analysis comparison of endoscopic papillary balloon dilatation and endoscopic sphincteropapillotomy. World J. Gastroenterol., 19, 3883-3891. 


\title{
В. І. ЛУПАЛЬЦОВ, А. І. ЯГНЮК, М. М. СКАЛІЙ, М. С. КОТОВЩИКОВ
}

Харківський національний медичний університет

\section{ПАНКРЕАТОБІЛІАРНА МАНОМЕТРІЯ У ПРОФІЛАКТИЦІ ПОСТМАНІПУЛЯЦЙНОГО ПАНКРЕАТИТУ ПІС.ЛЯ ЕНДОСКОПІЧНОЇ РЕТРОГРАДНОӤ ХОЛАНГІОПАНКРЕАТОГРАФІЇ}

\begin{abstract}
Мета роботи: зниження частоти виникнення гострого постманіпуляційного панкреатиту за рахунок використання панкреатобіліарної манометрії.

Матеріали і методи. Запропонований пристрій реєструє тиск у загальній панкреатичній протоці при виконанні ендоскопічної ретроградної холонгіопанкреатографії (ЕРХПГ), який в режимі реального часу, за допомогою графічної кривої, відображає тиск у панкреатичній протоці. Так, при різкому “піковому” підйомі кривої ситуацію розцінювали як підвищення тиску в панкреатичній протоці, що було показанням до введення контрасту з меншою об’ємною швидкістю і проведення протипанкреатичної терапії. Ця методика була використана у 128 пацієнтів з 2013 по 2018 рр.

Результати досліджень та їх обговорення. У хворих першої групи гострий постманіпуляційний панкреатит розвинувся у 21,8 \%, тоді як у пацієнтів другої групи, у яких профілактичні заходи гострого постманіпуляційного панкреатиту проводилися відразу після виявлення його ознак - у 6,3 \%. Клінічні симптоми гострого панкреатиту та амілолітична активність крові в групі, де застосовували методики манометричного контролю, спостерігалися рідше, ніж у групі порівняння.

При використанні розроблених методик манометричного контролю під час лікувально-діагностичних маніпуляцій на фатеровому сосочку достовірно знижується ризик виникнення гострого постманіпуляційного панкреатиту.
\end{abstract}

Ключові слова: гострий постманіпуляційний панкреатит; ендоскопічна ретроградна холангіопанкреатографія; панкреатобіліарний манометр.

\section{I. LUPALTSOV, A. I. YAGNYUK, N. N. SKALII, M. S. KOTOVSCHIKOV}

Kharkiv National Medical University

\section{PANCREATOBILIAR MANOMETRY IN PROPHYLAXIS OF POST-MANIPULATED PANCREATITIS AFTER ENDOSCOPIC RETROGRADE CHOLANGIOPANCREATOGRAPHY}

The aim of the work: to reduce the incidence of acute post-manipulative pancreatitis due to the use of pancreatobiliar manometry. Materials and Methods. A proposed device registers pressure in the common pancreatic duct when performing endoscopic retrograde cholangiopancreatography, which in real time, using a graphical curve, displays the pressure in the pancreatic duct. Thus, with a sharp "peak" rise in the curve, the situation was considered as an increase in pressure inside the pancreatic duct, which was an indication for the injection of contrast substance with a lower volume rate and anti-pancreatic therapy. This technique was used in 128 patients from 2013 to 2018.

Results and Discussion. In patients of group 1, acute post-manipulative pancreatitis developed in $21.8 \%$, while in patients of group 2 , in whom prophylactic measures of acute post-manipulative pancreatitis were carried out immediately after the detection of its manifestations - in $6.3 \%$. The clinical symptoms of acute pancreatitis and amylolytic blood activity were observed rarely in group, where the pressure control methods were used, than in the comparison group.

Key words: acute post-manipulative pancreatitis; endoscopic retrograde pancreatocholangiography; pancreatobiliar manometry. 